\title{
Difficulties and remedies on DEA environmental assessment
}

\author{
Toshiyuki Sueyoshi ${ }^{1}$ and Mika Goto ${ }^{2^{*}}$ [0
}

\author{
${ }^{*}$ Correspondence: \\ goto.m.af@m.titech.ac.jp \\ ${ }^{2}$ School of Environment \\ and Society, Tokyo Institute \\ of Technology, 2-12-1, \\ Ookayama, Meguro-ku, \\ Tokyo 152-8552, Japan \\ Full list of author information \\ is available at the end of the \\ article
}

\begin{abstract}
This study discusses a DEA approach for environmental assessment. The proposed approach examines a level of simultaneous achievement on economic prosperity and environmental protection, so measuring the level of "sustainability." DEA, standing for Data Envelopment Analysis, has been widely applied for performance assessment in the past five decades. A new type of methodology is referred to as "DEA environmental assessment," and it measures the performance of various organizations that use inputs to produce not only desirable outputs (e.g., electricity) but also undesirable outputs (e.g., $\mathrm{CO}_{2}$ emission). In this study, we discuss various methodological concerns by considering theoretical and empirical difficulties related to the use of DEA environmental assessment. These difficulties include (a) how to incorporate two separated (natural and managerial) disposability concepts into a unified framework of DEA environmental assessment, (b) how to reorganize unified disposability concepts in the proposed approach, (c) how to incorporate an occurrence of undesirable congestion (e.g., a line capacity limit on transmission) and desirable congestion (e.g., possible occurrence of green technology innovation) and (d) how to manage a data set that contains zero and negative values. It is easily expected that these explorations enhance the practicality of DEA environmental assessment.
\end{abstract}

Keywords: Environmental assessment, Sustainability, DEA

\section{Background}

Intergovernmental Panel on Climate Change (IPCC: http://ipcc.ch/index.htm), established within United Nations (UN) environmental program, reported the policy suggestion in April 2014 that it was necessary for us to reduce an amount of greenhouse gas (GHG) emissions, in particular $\mathrm{CO}_{2}$, by $40-70 \%$ (compared with 10 years ago) until 2050 and to reduce them at the level of almost zero by the end of this twenty-first century via shifting the current systems to energy-efficient ones. Otherwise, the IPCC has warned that the global warming and climate change will destroy our natural and socioeconomic systems. Consequently, we will face various risks (e.g., heat waves, droughts, floods, food crisis as well as damages to human, social and economic systems) on the earth.

To combat the global warming and climate change, we need to establish a "sustainable society" in which we can simultaneously attain both economic prosperity and environmental protection by coordinating social and economic systems. The sustainable development goals (SDGs) proposed by UN serves as important guidelines for achieving the sustainable 
society. Pursuing SDGs is closely linked to corporate management, because environmental problems have been long influencing not only our social and economic systems but also corporate operations in real business world because all industrial sectors need to consider their business strategies that adapt various regulation changes on industrial pollutions. The benefits from installing GHG reduction technologies or green technologies in private sectors range from intangible ones, such as improved public image as "green corporate citizen," to measurable ones such as reduced GHG emission levels. These benefits, for example, are derived from the promotion of green products and introduction of green supply chain systems in a firm. See Sueyoshi and Goto (2018, Chapter 14) for a detailed description of the history of industrial pollutions after the industrial revolution in the world.

In this study, a level of sustainability in various organizations is measured by their operational performance (as an economic prosperity measure) and environmental performance (as a pollution prevention measure) to achieve the sustainable society. A difficulty, associated with attaining a high level of sustainability, is that individuals who are interested in pollution prevention do not have a common practical methodology to assess the performance of organizations in terms of their operational and environmental achievements. Data Envelopment Analysis (DEA), proposed in this study, is one of such methodologies to assess the environmental performance and more broadly the level of sustainability. As an initial step for such a methodological development applied to corporate sustainability, this study is concerned with a description of difficulties and remedies incorporated in the proposed DEA environmental assessment. Considering these difficulties, this study proposes a new formulation for DEA environmental assessment.

Before discussing the proposed approach, it is important to specify the following important features of DEA. First, DEA measures a process of many organizations that utilize inputs to produce desirable outputs (e.g., electricity and services) by relatively comparing each one with others. Second, no functional form specification is necessary for DEA. Finally, we can solve it by linear programming so that DEA has a high computational capability. These are indeed methodological contributions of DEA.

However, DEA does not directly incorporate undesirable outputs (e.g., GHG and acid rain gas) in the computational framework because the directional vector (maximization) of desirable outputs and that (minimization) of undesirable outputs. Meanwhile, DEA environmental approach incorporates the existence of undesirable outputs in the computational framework. Thus, it can handle the three types of production factors (inputs, desirable and undesirable outputs) in the performance assessment. As a result, the DEA environmental assessment is computationally more complicated, but has more implications, than the original DEA. The methodological difficulties will be addressed in this study.

Here, it is important to note that Sueyoshi et al. (2017) and Sueyoshi and Goto (2018) have provided a list of more than 700 articles on DEA environmental assessment. See also Sueyoshi and Goto (2017) and Sueyoshi and Yuan (2018) that have discussed a recent trend of supply and demand in world energy along with its relationship with DEA applied to energy and environment. This study is a methodological extension of these previous studies. Note that various types of DEA applications can be found in the previous research efforts. Therefore, this study does not document any specific application, rather describing methodological strengths and drawbacks of DEA environmental assessment, all of which have not clearly addressed in these previous efforts. Such is a contribution of this study. 
An underlying philosophy discussed in this study is that the proposed DEA environmental assessment may serve as an important component for measuring corporate sustainability. Thus, the sustainability enhancement is essential for the management of many firms to survive in a global competition. Moreover, the concept is closely related to corporate social responsibility (CSR). ${ }^{1}$ In this study, environmental assessment is considered as part of CSR, so focusing on a business balance between economic prosperity and environmental protection.

Abbreviations used in this study are summarized as follows: CSCs: Complementary Slackness Conditions, CSR: Corporate Social Responsibility, DEA: Data Envelopment Analysis, DTS: Damages to Scale, DMU: Decision Making Unit, DC: Desirable Congestion, NR: Non-radial, RTS: Returns to Scale, UC: Undesirable Congestion, UEN: Unified Efficiency under Natural Disposability, UEM: Unified Efficiency under Managerial disposability, UEM(DC): Unfired Efficiency under Managerial Disposability and Desirable Congestion and URS: Unrestricted.

The remainder of this study is organized as follows. Section 2 describes methodological pitfalls. Section 3 discusses DEA non-radial models for environmental assessment. Section 4 describes a possible occurrence of undesirable and that of desirable congestions. Section 5 discusses how to incorporate the assumption that undesirable outputs are the by-products of desirable outputs into the proposed framework. Section 6 discusses how to handle zero and negative values. Sections from 3 to 6 document how the proposed new approach can overcome the pitfalls, discussed in this study, related to DEA environmental assessment. Section 7 concludes this study along with future extensions.

\section{Pitfalls}

\subsection{First pitfall: disposability concepts}

Disposability concepts Figure 1 depicts analytical structures for measuring a degree of unified (operational and environmental) efficiency that become an empirical basis for environmental assessment (Sueyoshi and Goto 2018). For our visual convenience, Fig. 1 considers the observed achievement, listed as K, for example, of a DMU that uses a single component of an input vector ( $x$ in the horizontal axis) to produce both a single component of a desirable output vector ( $g$ on the vertical axis) and that of an undesirable output vector ( $b$ on the vertical axis). The description is easily extendable to a more general case where multiple components of the three vectors are incorporated into the computational framework of the proposed DEA approach.

To explain an analytical implication of the unified efficiency measurement, let us consider that the achievement of the $k$ th DMU is specified as $\left(x_{i k}, g_{r k}, b_{f k}\right)$ in Fig. 1 , where the DMU is a specific organization to be evaluated. In the figure, possible performance enhancements, depicted in Fig. 1, are classified by the following four types of projections:

1. NW (Northwest) projections to enhance operational performance: If the $k$ th DMU is inefficient in operational performance, then it needs to improve the operational efficiency by increasing the amount of a desirable output and/or decreasing the amount of an input. A possible projection can be found on $\{B\}$ as such an example. A piece-

${ }^{1}$ See previous studies on DEA applied to CSR including Belu (2009), Berber et al. (2011), Lundgren and Zhou (2017), McWilliams et al. (2016), Pérez-Gladish et al. (2013), Sueyoshi et al. (2010), Vitaliano and Stella (2006), Wang et al. (2014) as well as Yang $(2016,2017)$. 


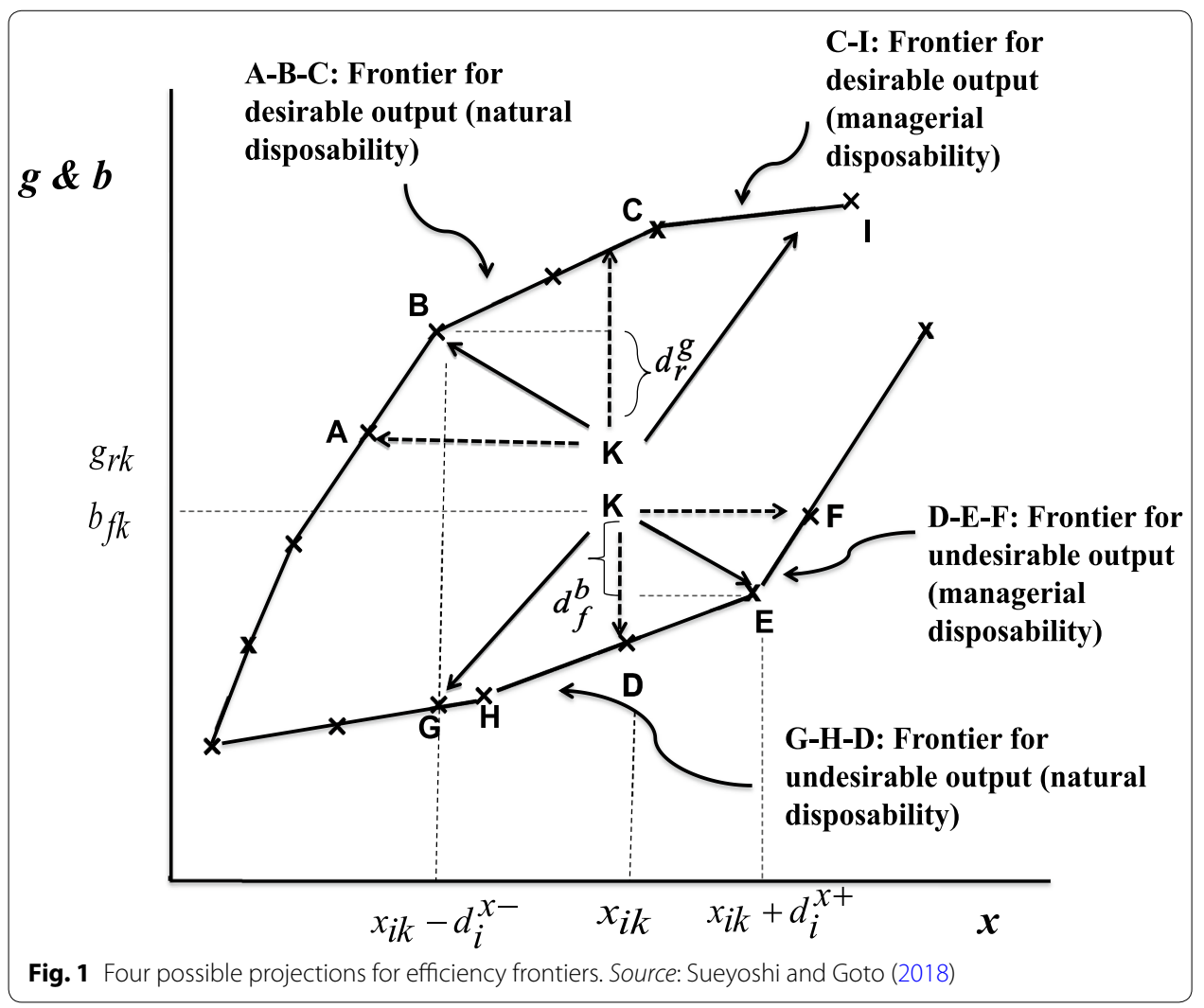

wise linear contour line $(\{A\}-\{B\}-\{C\})$ indicates an efficiency frontier where $\{K\}$ needs to be projected. The direction of such possible projections toward $\{A\}-\{B\}-\{C\}$ is expressed by both the sign of an input-related slack and that of a desirable outputrelated slack, along with the direction of a unified inefficiency measure, as depicted in Fig. 1. This type of projections belongs to "NW" which indicates the enhancement of operational performance. Such projections serve a basis for enhancing the level of operational efficiency within conventional DEA based upon modern production economics. The projection looks for the best strategy to increase a desirable output under "economic stagnation." The projection also naturally implies a decrease in an undesirable output (i.e., industrial pollution), so indicating the concept of "natural disposability."

2. NE (Northeast) projections to enhance operational performance: The projection of NE has been excluded from the conventional use of DEA until now. The proposed DEA assessment incorporates the type of projections toward NE on the piece-wise linear contour line $(\{C\}-\{I\})$, along with an increase in both an input and a desirable output, as depicted in the right-hand side of Fig. 1. The type of projection for enhancing the level of operational performance implies "economic growth" of the $k$ th DMU. The growth is usually associated with various industrial pollutions. Therefore, this study must consider how to make an industrial balance between them. We clearly 
understand that it is not easily attainable such a balance in reality because it needs to make a considerable effort, so being referred to as "managerial disposability." This type of performance enhancement has been never discussed in conventional DEA. The contribution of DEA environmental assessment is that it incorporates the type of projections, or corporate efforts, toward NE.

3. SW (Southwest) projections to enhance environmental performance: Shifting our interest from operational to environmental performance enhancement, Fig. 1 visually describes a projection to enhance the amount of the input (on a horizontal coordinate) and the undesirable output (on a vertical coordinate) whose type of projections direct toward SW along with an input decrease. One of such directions is the projection from $\{K\}$ to $\{G\}$ in Fig. 1. Here, $\{G\}$ stands for the performance on an efficiency frontier for an undesirable output and an input. The piece-wise linear contour line $(\{G\}-\{H\}-\{D\})$ indicates such a frontier for unified efficiency. This type of projections, occurring with an input decrease, naturally decreases an amount of the undesirable output. Thus, this study considers the projection as "natural disposability" to enhance environmental performance.

4. SE (Southeast) projections to enhance environmental performance: The type of projections from $\{K\}$ to $\{E\}$ indicates an opposite case of the $S W$ projection, but indicating an enhancement of environmental performance. In the case, an efficiency frontier for the $k$ th $D M U$ can be found on the piece-wise linear contour line $(\{D\}-$ $\{E\}-\{F\})$ where the DMU needs to reduce the amount of the undesirable output by green technology and/or managerial effort, along with an input increase. This type of projections, under managerial disposability, has been never explored in the previous DEA studies.

Axiomatic Expression To describe the four possible projections more clearly, let us consider $X \in R_{+}^{m}$ as an input vector with $m$ components, $G \in R_{+}^{s}$ as a desirable output vector with $s$ components and $B \in R_{+}^{h}$ as an undesirable output vector with $h$ components. In these column vectors, the subscript $(j)$ is used to indicate the $j$ th DMU, whose vector components are all strictly positive.

Using an axiomatic expression, this study specifies unified production and pollution possibility sets to express natural $(N)$ and that for managerial $(M)$ disposability by the two types of output vectors and an input vector, respectively, as follows:

$$
\begin{gathered}
P_{v}^{N}(X)=\left\{(G, B): G \leq \sum_{j=1}^{n} G_{j} \lambda_{j}, B \geq \sum_{j=1}^{n} B_{j} \lambda_{j}, X \geq \sum_{j=1}^{n} X_{j} \lambda_{j}, \sum_{j=1}^{n} \lambda_{j}=1 \& \lambda_{j} \geq 0(j=1, \ldots, n)\right\} \text { and } \\
P_{v}^{M}(X)=\left\{(G, B): G \leq \sum_{j=1}^{n} G_{j} \lambda_{j}, B \geq \sum_{j=1}^{n} B_{j} \lambda_{j}, X \leq \sum_{j=1}^{n} X_{j} \lambda_{j}, \sum_{j=1}^{n} \lambda_{j}=1 \& \lambda_{j} \geq 0(j=1, \ldots, n)\right\} .
\end{gathered}
$$

$P_{v}^{N}(X)$ stands for a production and pollution possibility set under natural disposability. Meanwhile, $P_{v}^{M}(X)$ is that of managerial disposability. The subscript $(v)$ 
stands for variable Returns to Scale (RTS) ${ }^{2}$ or variable Damages to Scale (DTS) ${ }^{3}$ because the constraint $\left(\sum_{j=1}^{n} \lambda_{j}=1\right)$ is incorporated in the two axiomatic expres-

sions. See Sueyoshi (2017) for the similar concept for DTS. The difference between the two disposability concepts is that the production technology under natural disposability, or $P_{v}^{N}(X)$, has $X \geq \sum_{j=1}^{n} X_{j} \lambda_{j}$, implying that a DMU can attain an efficiency frontier by reducing a directional vector of inputs.

Besides the above difference, the two disposability concepts have a common feature that both have $G \leq \sum_{j=1}^{n} G_{j} \lambda_{j}$ and $B \geq \sum_{j=1}^{n} B_{j} \lambda_{j}$ in these axiomatic expressions. These conditions intuitively appeal to us because an efficiency frontier for desirable outputs should locate above or on all observations, while that of undesirable outputs should locate below or on these observations.

\subsection{Second pitfall: disposability unification}

Disposability unification An important aspect of DEA environmental assessment is that it separates outputs into desirable and undesirable categories. The output separation cannot be found in the conventional framework of DEA. The two types of outputs are different in terms of their analytical structures (e.g., the direction of these vectors). A problem to be overcome after the output separation is that it is necessary for us to consider conceptual frameworks and these related formulations for unifying desirable and undesirable outputs. Figure 2 depicts the unification process from I to III, all of which are incorporated in the proposed approach for environmental assessment. For our visual description, the figure depicts a case of a single component of three production factors. An extension to multiple components is possible in mathematical models to be discussed in this study.

First, Stage I consist of two substages: (A) and (B). Stage I indicates the production relationship between an input $(x)$ and a desirable output $(g)$ under the assumption that all DMUs produce a same amount of undesirable output (b). A Production Possibility Set (PrPS) locates below a piece-wise linear convex curve, depicting an efficiency frontier $\left(E F_{g}\right)$ in the $x-g$ space. Stage I has the other substage (B). Meanwhile, a pollution possibility set (PoPS) locates above the piece-wise linear concave curve that expresses an

\footnotetext{
2 To describe RTS in DEA environmental assessment, we measure the degree of scale elasticity $\left(e_{g}\right)$ between an input $(x)$ and a desirable output $(g)$. Let us consider a simple case in which a supporting hyperplane is mathematically expressed by $v x-u g+w b+\sigma=0$. For our descriptive convenience, all production factors consist of a single component. Here, the undesirable output $(b)$ is additionally incorporated in the equation. The symbols $(v, u$ and $w)$ are parameters of a supporting hyperplane, indicating the degree of each slope regarding the supporting hyperplane. All the parameters are positive in their signs. An exception is $\sigma$ that is unrestricted in the sign and it indicates an intercept of the supporting hyperplane. Using the supporting hyperplane, we obtain $\frac{d g}{d x}=\frac{v}{u}$ and $\frac{g}{x}=\frac{v}{u}+\frac{\sigma+w b}{u x}$. Consequently, the scale elasticity between $g$ and $x$ is measured by $e_{g}=\left(\frac{\mathrm{d} g}{\mathrm{~d} x}\right) /\left(\frac{g}{x}\right)=1 /\left(1+\frac{\sigma+w b}{v x}\right)$. The degree of the scale elasticity $\left(e_{g}\right)$ related to a desirable output is classified by $\sigma$ and $w$ by the following rule: (a) $e_{g}>1 \leftrightarrow$ Increasing RTS $\leftrightarrow \sigma+w b<0$, (b) $e_{g}=1 \leftrightarrow$ Constant RTS $\leftrightarrow \sigma+w b=0$ and (c) $e_{g}<1 \leftrightarrow$ Decreasing RTS $\leftrightarrow \sigma+w b>0$.

3 To discuss DTS, let us consider scale elasticity $\left(e_{b}\right)$ between an input and an undesirable output. We consider that a supporting hyperplane is $-v x-u g+w b+\sigma=0$ where the three production factors consist of a single component. Then, we have $\frac{d b}{d x}=\frac{v}{w}$ and $\frac{b}{x}=\frac{v}{w}-\frac{\sigma-u g}{w x}$. Consequently, the scale elasticity related to an undesirable output is measured by $e_{b}=\left(\frac{d b}{d x}\right) /\left(\frac{b}{x}\right)=1 /\left(1-\frac{\sigma-u g}{v x}\right)$. The scale elasticity $\left(e_{b}\right)$ related to an input and an undesirable output is determined by $\sigma$ and $u$ as follows: (a) $e_{b}>1 \leftrightarrow$ Increasing DTS $\leftrightarrow \sigma-u g>0$, (b) $e_{b}=1 \leftrightarrow$ Constant DTS $\leftrightarrow \sigma-u g=0$ and (c) $e_{b}<1 \leftrightarrow$ Decreasing DTS $\leftrightarrow \sigma-u g<0$.
} 


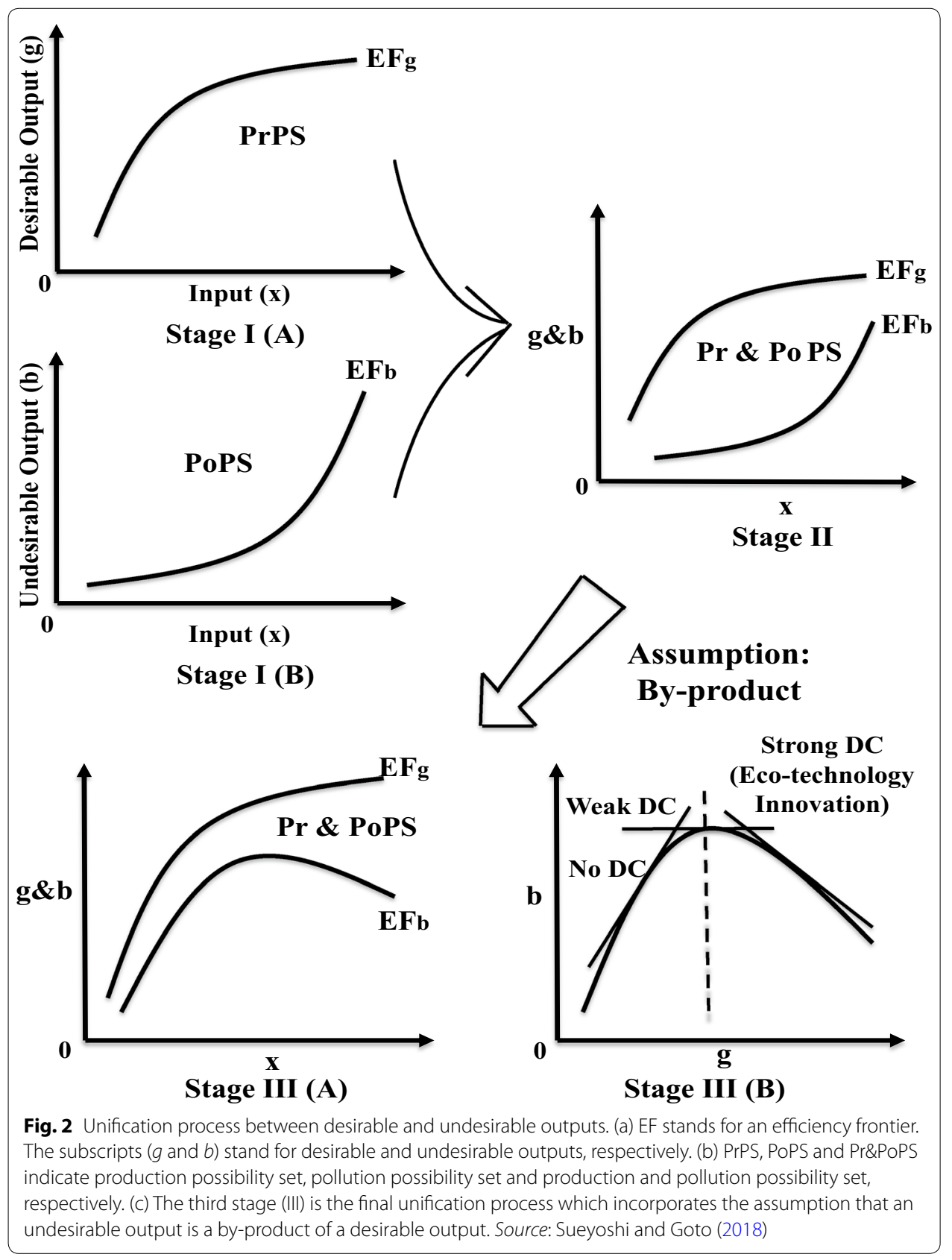

efficiency frontier $\left(E F_{b}\right)$ in the $x-b$ space under the assumption that they produce a same amount of a desirable output $(g)$. An important feature of Stage I is that the production possibility set (A) is independent from the pollution possibility set (B). This assumption is slightly unrealistic because both are relatively related to each other.

To unify the two sets related to Stage I, Stage II combines them in a unified framework as depicted in Fig. 2 whose coordinates indicate $x$ and $g \mathcal{E} b$, respectively. The unification process makes it possible to identify a production and pollution possibility set (Pr\&PoPS) between PrPS and PoPS, locating between the two efficiency frontiers ( $E F_{g}$ and $E F_{b}$ ). All DMUs locate within Pr\&PoPS between the production and pollution possibility sets of Stage I (A) and (B). 


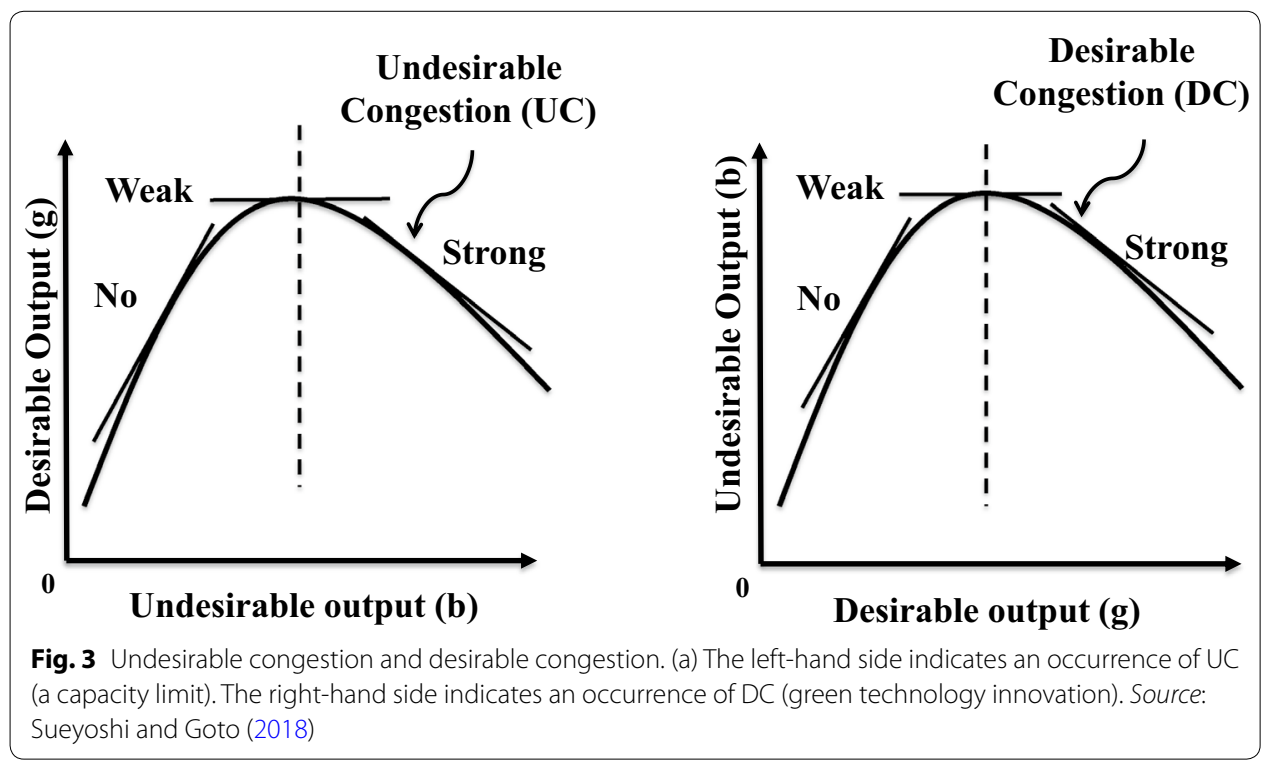

Stage III is the final unification process which incorporates an assumption that "undesirable outputs are by-product of desirable outputs." The assumption acceptable, but the assumption changes the two efficiency frontiers $\left(E F_{g}\right.$ and $\left.E F_{b}\right)$ to be shaped by two convex forms, as depicted in Stage III (A) of Fig. 2. Here, the efficiency frontier $\left(E F_{g}\right)$ should have an increasing trend along with an input increase. However, the efficiency frontier $\left(E F_{b}\right)$ should have an increasing and decreasing trend due to green technology innovation for reducing an amount of pollution $(b)$. Both curves should have piece-wise linear convex forms because of the by-product assumption. Thus, they are structurally different from those of the first and second stages. Stage III (B) depicts such a relationship between $g$ on the horizontal axis and $b$ on the vertical axis.

\subsection{Third pitfall: undesirable congestion (UC) and desirable congestion (DC)}

$U C$ and $D C$ Figure 3 visually specifies the importance of congestion on undesirable outputs in DEA environmental assessment. The concept of congestion is separated into Undesirable Congestion (UC) and Desirable Congestion (DC) in the proposed environmental assessment. Figure 3 depicts differences between UC and DC. The left-hand side of Fig. 3 exhibits the three types of UC in a space of an undesirable output $(b)$ on the horizontal axis and a desirable output $(g)$ on the vertical axis. The right-hand side of Fig. 3 exhibits the three types of DC in a space of a desirable output $(g)$ on a horizontal axis and an undesirable output $(b)$ on the vertical axis.

The analytical importance of such an occurrence of UC is that it is characterized by a supporting hyperplane. For example, as depicted in the left-hand side of Fig. 3, the negative slope of a supporting line indicates an occurrence of "strong UC." For example, the occurrence indicates a capacity limit on part or whole of a production facility (e.g., transmission in the electric power industry or transportation in the petroleum industry). In contrast, a positive slope implies an opposite case (i.e., no occurrence of UC), so being "no UC." An occurrence of "weak UC" is identified between strong UC and no UC, so being "weak UC." 
The occurrence of UC is problematic in many energy industries, as discussed above. Acknowledging the importance of conventional research on UC, however, this study is interested in sustainability development by enhancing the level of economic prosperity and reducing the amount of industrial pollution. Therefore, we pay attention to a possible occurrence of DC, hereafter, because the occurrence indicates green technology innovation so that it can enhance the level of social or corporate sustainability.

Stage III (B) of Fig. 2 exhibits such a possible occurrence of DC (green technology innovation) of Fig. 3. The negative slope of a supporting hyperplane indicates an occurrence of "strong DC," or green technology innovation for reducing an undesirable output (e.g., industrial pollution). In contrast, a positive slope implies an opposite case (i.e., no occurrence of DC), so being "no DC." An occurrence of "weak DC" is identified between strong and no DC, so being "weak DC."

\subsection{Fourth pitfall: zero and negative values}

Zero and negative values Another difficulty in applying DEA to energy and environment is that it cannot handle zero and negative value in a data set. Most of the previous studies assume that all observations are strictly positive. The assumption is often problematic in reality, because many energy firms (e.g., oil and gas companies) often produce negative net incomes. Many financial performance measures of these firms, expressing economic prosperity, may become negative in their signs.

This study discusses the property of "translation invariance" under DC. Our discussion is applicable to UC. The property implies that an efficiency measure should be not influenced even if production factors are shifted toward a same direction by adding or subtracting a specific real number. The property makes it possible that we can evaluate the performance of entities, whose production and envelopment factors contain zero and/or negative values. See Sect. 3 that discusses the property of translation invariance.

\section{Non-radial models}

The unified (operational and environment) efficiency is measured by the three different approaches, using radial, non-radial and intermediate models, respectively. This study uses the non-radial models because they can handle the zero and negative values in a data set by the property of "translation invariance" in the analytical frameworks.

Nomenclatures used in this study are summarized as follows: $X$ : A column vector of $m$ inputs, US: United States, G: A column vector of $s$ desirable outputs, $B$ : A column vector of $h$ undesirable outputs, $d_{i}^{x}$ : An unknown slack variable of the $i$ th input, $d_{r}^{g}$ : An unknown slack variable of the $r$ th desirable output, $d_{f}^{b}$ : An unknown slack variable of the

$f$ th undesirable output, $\lambda$ : An unknown column vector of intensity (or structural) variables, $R_{i}^{x}$ : A data range related to the $i$ th input, $R_{r}^{g}$ : A data range related to the $r$ th desirable output, $R_{f}^{b}$ : A data range related to the $f$ th undesirable output, $v_{i}$ : A dual variable of

the $i$ th input, $u_{r}$ : A dual variable of the $r$ th desirable output, $w_{f}$ : A dual variable of the $f$ th undesirable output, $\sigma$ : A dual variable to indicate the intercept of a supporting 
hyperplane on a production and pollution possibility set and $\varepsilon$ : a prescribed small number to control the magnitude of unified efficiency.

This study specifies the following three types of data ranges $(R)$ according to the upper and lower bounds of production factors:

$$
\begin{aligned}
& R_{i}^{x}=(m+s+h)^{-1}\left(\max \left\{x_{i j} \mid j=1, \ldots, n\right\}-\min \left\{x_{i j} \mid j=1, \ldots, n\right\}\right)^{-1}, \\
& R_{r}^{g}=(m+s+h)^{-1}\left(\max \left\{g_{r j} \mid j=1, \ldots, n\right\}-\min \left\{g_{r j} \mid j=1, \ldots, n\right\}\right)^{-1} \text { and } \\
& R_{f}^{b}=(m+s+h)^{-1}\left(\max \left\{b_{f j} \mid n=1, \ldots, n\right\}-\min \left\{b_{f j} \mid n=1, \ldots, n\right\}\right)^{-1} .
\end{aligned}
$$

Under natural disposability The non-radial model under natural disposability $(N)$ to measure the unified efficiency (UEN), combining desirable and undesirable outputs of the $k$ th DMU, is formulated by the following model (e.g., Sueyoshi and Goto 2018):

$$
\begin{aligned}
\text { Maximize } & \varepsilon\left(\sum_{i=1}^{m} R_{i}^{x} d_{i}^{x-}+\sum_{r=1}^{s} R_{r}^{g} d_{r}^{g}+\sum_{f=1}^{h} R_{f}^{b} d_{f}^{b}\right) \\
\text { s.t. } & \sum_{j=1}^{n} x_{i j} \lambda_{j}+d_{i}^{x-}=x_{i k} \quad(i=1, \ldots, m), \\
& \sum_{j=1}^{n} g_{r j} \lambda_{j}-d_{r}^{g}=g_{r k} \quad(r=1, \ldots, s), \\
& \sum_{j=1}^{n} b_{f j} \lambda_{j}+d_{f}^{b}=b_{f k} \quad(f=1, \ldots, h), \\
& \sum_{j=1}^{n} \lambda_{j}=1, \\
& \lambda_{j} \geq 0 \quad(j=1, \ldots, n), \quad d_{i}^{x-} \geq 0 \quad(i=1, \ldots, m), \\
& d_{r}^{g} \geq 0 \quad(r=1, \ldots, s) \& d_{f}^{b} \geq 0 \quad(f=1, \ldots, h) .
\end{aligned}
$$

The right-hand side of Model (1) indicates an observed performance of the $k$ th DMU in terms of the three production factors. Meanwhile, the left-hand side expresses a composite (or ideal) performance on these factors regarding DMUs on an efficiency frontier. Model (1) relatively compares their performance measures to estimate a unified efficiency score. Model (1) incorporates the three types of slacks for inefficiency measurement. Among them, the model considers only deviations $+d_{i}^{x-}$ $(i=1, \ldots, m)$ related to inputs in order to attain the status of natural disposability.

The unified efficiency $\left(\mathrm{UEN}_{v}^{\mathrm{NR}}\right.$ ) of the $k$ th DMU under natural disposability and variable RTS is measured by

$$
\mathrm{UEN}_{v}^{\mathrm{NR}}=1-\varepsilon\left(\sum_{i=1}^{m} R_{i}^{x} d_{i}^{x-*}+\sum_{r=1}^{s} R_{r}^{g} d_{r}^{g *}+\sum_{f=1}^{h} R_{f}^{b} d_{f}^{b *}\right),
$$

where all slack variables are determined on the optimality of Model (1). The equation within the parenthesis, obtained from the optimality of Model (1), indicates the level of 
unified inefficiency under natural disposability. The unified efficiency is obtained by subtracting the level of inefficiency from unity, as formulated in Eq. (2).

Model (1) has the following dual formulation:

$$
\begin{aligned}
& \operatorname{Minimize} \sum_{i=1}^{m} v_{i} x_{i k}-\sum_{r=1}^{s} u_{r} g_{r k}+\sum_{f=1}^{h} w_{f} b_{f k}+\sigma \\
& \text { s.t. } \sum_{i=1}^{m} v_{i} x_{i j}-\sum_{r=1}^{s} u_{r} g_{r j}+\sum_{f=1}^{h} w_{f} b_{f j}+\sigma \geq 0 \quad(j=1, \ldots, n) \text {, } \\
& v_{i} \geq \varepsilon R_{i}^{x} \quad(i=1, \ldots, m), \\
& u_{r} \geq \varepsilon R_{r}^{g} \quad(r=1, \ldots, s), \\
& w_{f} \geq \varepsilon R_{f}^{b} \quad(f=1, \ldots, h), \\
& \sigma \text { : URS, }
\end{aligned}
$$

where $v_{i}(i=1, \ldots, m), u_{r}(r=1, \ldots, s)$ and $w_{f}(f=1, \ldots, h)$ are all dual variables related to the first, second and third groups of constraints in Model (1). The dual variable $(\sigma)$ is obtained from the fourth equation of Model (1). An important feature of Model (3) is that all dual variables are positive so that all the production factors are fully utilized in the proposed environmental assessment.

Under managerial disposability Shifting our description from natural disposability to managerial disposability, this chapter can measure the unified efficiency under managerial disposability $(M)$ where environmental performance is the first priority and operational performance is the second priority. The priority has of managerial disposability an opposite priority to that of natural disposability.

To measure the unified efficiency $\left(\mathrm{UEM}_{v}^{\mathrm{NR}}\right)$ of the $k$ th DMU under managerial disposability, the research of Sueyoshi and Goto (2018) has proposed the following non-radial model in an augmented form:

$$
\begin{aligned}
\text { Maximize } & \varepsilon\left(\sum_{i=1}^{m} R_{i}^{x} d_{i}^{x+}+\sum_{r=1}^{s} R_{r}^{g} d_{r}^{g}+\sum_{f=1}^{h} R_{f}^{b} d_{f}^{b}\right) \\
\text { s.t. } & \sum_{j=1}^{n} x_{i j} \lambda_{j}-d_{i}^{x+}=x_{i k} \quad(i=1, \ldots, m), \\
& \sum_{j=1}^{n} g_{r j} \lambda_{j}-d_{r}^{g}=g_{r k} \quad(r=1, \ldots, s), \\
& \sum_{j=1}^{n} b_{f j} \lambda_{j}+d_{f}^{b}=b_{f k} \quad(f=1, \ldots, h), \\
& \sum_{j=1}^{n} \lambda_{j}=1, \\
& \lambda_{j} \geq 0 \quad(j=1, \ldots, n), \quad d_{i}^{x+} \geq 0 \quad(i=1, \ldots, m), \\
& d_{r}^{g} \geq 0 \quad(r=1, \ldots, s) \& d_{f}^{b} \geq 0 \quad(f=1, \ldots, h) .
\end{aligned}
$$


Model (4) considers only deviations $-d_{i}^{x+}(i=1, \ldots, m)$ related to inputs in order to attain the status of managerial disposability.

The unified efficiency $\left(\mathrm{UEM}_{v}^{\mathrm{NR}}\right)$ of the $k$ th DMU under managerial disposability and variable DTS is measured by

$$
\mathrm{UEM}_{v}^{\mathrm{NR}}=1-\varepsilon\left(\sum_{i=1}^{m} R_{i}^{x} d_{i}^{x+*}+\sum_{r=1}^{s} R_{r}^{g} d_{r}^{g *}+\sum_{f=1}^{h} R_{f}^{b} d_{f}^{b *}\right)
$$

where all slack variables are determined on the optimality of Model (4). The equation within the parenthesis, obtained from the optimality of Model (4), indicates the level of unified inefficiency under managerial disposability. The unified efficiency is obtained by subtracting the level of inefficiency from unity, as formulated in Eq. (5).

Model (4) has the following dual formulation:

$$
\begin{aligned}
& \text { Minimize }-\sum_{i=1}^{m} v_{i} x_{i k}-\sum_{r=1}^{s} u_{r} g_{r k}+\sum_{f=1}^{h} w_{f} b_{f k}+\sigma \\
& \text { s.t. } \quad-\sum_{i=1}^{m} v_{i} x_{i j}-\sum_{r=1}^{s} u_{r} g_{r j}+\sum_{f=1}^{h} w_{f} b_{f j}+\sigma \geq 0 \quad(j=1, \ldots, n) \text {, } \\
& v_{i} \geq \varepsilon R_{i}^{x} \quad(i=1, \ldots, m), \\
& u_{r} \geq \varepsilon R_{r}^{g} \quad(r=1, \ldots, s), \\
& w_{f} \geq \varepsilon R_{f}^{b} \quad(f=1, \ldots, h), \\
& \sigma: \mathrm{URS}
\end{aligned}
$$

where $v_{i}(i=1, \ldots, m), u_{r}(r=1, \ldots, s)$ and $w_{f}(f=1, \ldots, h)$ are all dual variables related to the first, second and third groups of constraints in Model (4). The dual variable $(\sigma)$ is obtained from the fourth equation of Model (4).

At the end of this section, it is important to confirm that Models (1) and (4) solve the first pitfall related to DEA environmental assessment.

\section{Incorporation of UC and DC}

\subsection{A possible occurrence of undesirable congestion (UC)}

As depicted in the left-hand side of Fig. 3, this study may identify a possible occurrence of UC under natural disposability. To examine the occurrence, we use the following model that maintains equality constraints (so, no slack variable) on undesirable outputs: 


$$
\begin{aligned}
\text { Maximize } & \varepsilon\left(\sum_{i=1}^{m} R_{i}^{x-} d_{i}^{x-}+\sum_{r=1}^{s} R_{r}^{g} d_{r}^{g}\right) \\
\text { s.t. } & \sum_{j=1}^{n} x_{i j} \lambda_{j}+d_{i}^{x-}=x_{i k} \quad(i=1, \ldots, m), \\
& \sum_{j=1}^{n} g_{r j} \lambda_{j}-d_{r}^{g}=g_{r k} \quad(r=1, \ldots, s), \\
& \sum_{j=1}^{n} b_{f j} \lambda_{j} \quad=b_{f k} \quad(f=1, \ldots, h), \\
& \sum_{j=1}^{n} \lambda_{j}=1, \\
& \lambda_{j} \geq 0 \quad(j=1, \ldots, n), \\
& d_{i}^{x-} \geq 0 \quad(i=1, \ldots, m) \& d_{r}^{g} \geq 0 \quad(r=1, \ldots, s) .
\end{aligned}
$$

Model (7) drops slack variables related to undesirable outputs $(B)$ so that they are considered as equality constraints. The other constraints regarding inputs and desirable outputs are considered as inequality because they have slack variables in Model (7).

Model (7) has the following dual formulation:

$$
\begin{aligned}
& \text { Minimize } \sum_{i=1}^{m} v_{i} x_{i k}-\sum_{r=1}^{s} u_{r} g_{r k}+\sum_{f=1}^{h} w_{f} b_{f k}+\sigma \\
& \text { s.t. } \quad \sum_{i=1}^{m} v_{i} x_{i j}-\sum_{r=1}^{s} u_{r} g_{r j}+\sum_{f=1}^{h} w_{f} b_{f j}+\sigma \geq 0 \quad(j=1, \ldots, n) \text {, } \\
& v_{i} \geq \varepsilon R_{i}^{x} \quad(i=1, \ldots, m), \\
& u_{r} \geq \varepsilon R_{r}^{g} \quad(r=1, \ldots, s), \\
& w_{f}: \text { URS } \quad(f=1, \ldots, h) \& \\
& \sigma \text { : URS. }
\end{aligned}
$$

An important feature of Model (8) is that the dual variables ( $w_{f}:$ URS for $\left.f=1, \ldots, h\right)$ are unrestricted in their signs because the constraints on undesirable outputs are expressed by equality (i.e., no slack) in Model (7).

A unified efficiency measure of the $k$ th DMU under natural disposability becomes

$$
\begin{aligned}
\operatorname{UEN}(\mathrm{UC})_{v}^{N R *} & =1-\left[\varepsilon\left(\sum_{i=1}^{m} R_{i}^{x} d_{i}^{x-*}+\sum_{r=1}^{s} R_{r}^{g} d_{r}^{g *}\right)\right] \\
& =1-\varepsilon\left[\sum_{i=1}^{m} v_{i}^{*} x_{i k}-\sum_{r=1}^{s} u_{r}^{*} g_{r k}+\sum_{f=1}^{h} w_{f}^{*} b_{f k}+\sigma^{*}\right],
\end{aligned}
$$

which incorporates a possible occurrence of UC. All variables used in Eq. (9) are determined on the optimality of Models (7) and (8). The equation within the parenthesis, 
obtained from the optimal objective value of Models (7) and (8), indicates the level of unified inefficiency under natural disposability. The unified efficiency is obtained by subtracting the level of inefficiency from unity as specified in Eq. (9).

\subsection{A possible occurrence of desirable congestion (DC)}

As depicted in the right-hand side of Fig. 3, this study can identify an occurrence of Desirable Congestion (DC) under managerial disposability. To examine the occurrence, we use the following model, returning to Model (4), which maintains equality constraints (so, no slack variable) on desirable outputs:

$$
\begin{aligned}
\text { Maximize } & \varepsilon\left(\sum_{i=1}^{m} R_{i}^{x} d_{i}^{x+}+\sum_{f=1}^{h} R_{f}^{b} d_{f}^{b}\right) \\
\text { s.t. } & \sum_{j=1}^{n} x_{i j} \lambda_{j}-d_{i}^{x+}=x_{i k} \quad(i=1, \ldots, m), \\
& \sum_{j=1}^{n} g_{r j} \lambda_{j} \quad=g_{r k} \quad(r=1, \ldots, s), \\
& \sum_{j=1}^{n} b_{f j} \lambda_{j}+d_{f}^{b}=b_{f k} \quad(f=1, \ldots, h), \\
& \sum_{j=1}^{n} \lambda_{j}=1, \\
& \lambda_{j} \geq 0 \quad(j=1, \ldots, n), \quad d_{i}^{x+} \geq 0 \quad(i=1, \ldots, m) \& \\
& d_{f}^{b} \geq 0 \quad(f=1, . ., h) .
\end{aligned}
$$

Model (10) drops slack variables related to desirable outputs so that they are considered as equality constraints. The other groups of constraints on inputs and undesirable outputs do not have slacks so that they can be considered as inequality constraints.

A unified efficiency score, or UEM(DC) ${ }_{v}^{\mathrm{NR} *}$, of the $k$ th DMU under managerial disposability becomes

$$
\mathrm{UEM}(\mathrm{DC})_{v}^{\mathrm{NR} *}=1-\varepsilon\left(\sum_{i=1}^{m} R_{i}^{x} d_{i}^{x+*}+\sum_{f=1}^{h} R_{f}^{b} d_{f}^{b *}\right),
$$

where all variables are determined on the optimality of Model (10). The equation within the parenthesis, obtained from the optimality of Model (10), indicates the level of unified inefficiency under managerial disposability. The unified efficiency, along with a possible occurrence of DC, is obtained by subtracting the level of inefficiency from unity.

Model (10) has the following dual formulation: 


$$
\begin{aligned}
& \text { Minimize } \quad-\sum_{i=1}^{m} v_{i} x_{i k}+\sum_{r=1}^{s} u_{r} g_{r k}+\sum_{f=1}^{h} w_{f} b_{f k}+\sigma \\
& \text { s.t. } \quad-\sum_{i=1}^{m} v_{i} x_{i j}+\sum_{r=1}^{s} u_{r} g_{r j}+\sum_{f=1}^{h} w_{f} b_{f j}+\sigma \geq 0 \quad(j=1, \ldots, n) \text {, } \\
& v_{i} \geq \varepsilon R_{i}^{x} \\
& u_{r} \text { : URS } \\
& (i=1, \ldots, m), \\
& (r=1, \ldots, s) \text {, } \\
& w_{f} \geq \varepsilon R_{f}^{b} \\
& (f=1, \ldots, h) \&
\end{aligned}
$$

An important feature of Model (12) is that the dual variables $\left(u_{r}\right.$ : URS for $\left.r=1, \ldots, s\right)$ are unrestricted in these signs because Model (10) does not have slacks related to desirable outputs.

At the end of this section, it is important to note that the formulations discussed in this section overcome the third pitfall (i.e., UC and DC) related to DEA environment assessment.

\section{New approach: managerial unification under by-product assumption}

The two figures at the bottom of Fig. 2 depict the final structure of Stage III for disposability unification under the assumption that "undesirable outputs are the by-productions of desirable outputs." The assumption may be acceptable because undesirable outputs exist only if DMUs produce desirable outputs. Here, the important concern to be discussed is that the by-product assumption indicates that the efficiency frontier $\left(\mathrm{EF}_{g}\right)$ on desirable outputs increases along with an input increase as depicted in Stage III (A). Meanwhile, the efficiency frontier $\left(\mathrm{EF}_{b}\right)$ on undesirable output increases along with the input increase because of the by-product assumption. The increasing trend changes to decrease after a DMU accepts green technology. Such an introduction may have an increasing and decreasing trend as found in Stage III (B).

To formulate Stage III (B), this study reorganizes Model (10) to produce the following new model under managerial disposability:

$$
\begin{aligned}
\text { Maximize } & \varepsilon\left(\sum_{i=1}^{m} R_{i}^{x} d_{i}^{x+}+\sum_{f=1}^{h} R_{f}^{b} d_{f}^{b}\right) \\
\text { s.t. } & \sum_{j=1}^{n} x_{i j} \lambda_{j}-d_{i}^{x+}=x_{i k} \quad(i=1, \ldots, m), \\
& \sum_{j=1}^{n} g_{r j} \lambda_{j} \quad=g_{r k} \quad(r=1, \ldots, s), \\
& \sum_{j=1}^{n} b_{f j} \lambda_{j}-d_{f}^{b}=b_{f k} \quad(f=1, \ldots, h), \\
& \sum_{j=1}^{n} \lambda_{j}=1, \\
& \lambda_{j} \geq 0 \quad(j=1, \ldots, n), \quad d_{i}^{x+} \geq 0 \quad(i=1, \ldots, m) \& \\
& d_{f}^{b} \geq 0 \quad(f=1, \ldots, h) .
\end{aligned}
$$


An important feature of Model (13) to be noted is that it changes the sign of $+d_{f}^{b}$ in Model (10) to $-d_{f}^{b}$ in Model (13). The rationale is because of the assumption on byproduct. Both the efficiency frontiers $\left(\mathrm{EF}_{g}\right.$ and $\left.\mathrm{EF}_{b}\right)$ have similar convex shapes as depicted in Stage III (A) of Fig. 2. A production and pollution possibility set locates between them. The other important feature is that it is formulated under managerial disposability, as depicted in Stage III (B). We are interested in innovation for green technology at DMUs. Thus, it is necessary for us to discuss DC in the framework of Model (13) only under managerial disposability.

A unified efficiency score of the $k$ th DMU under managerial disposability becomes Eq. (11), or UEM(DC) $)_{v}^{\mathrm{NR} *}=1-\varepsilon\left(\sum_{i=1}^{m} R_{i}^{x} d_{i}^{x+*}+\sum_{f=1}^{h} R_{f}^{b} d_{f}^{b *}\right)$, where all variables are determined on the optimality of Model (13). The equation within the parenthesis, obtained from the optimality of Model (13), indicates the level of unified inefficiency under managerial disposability along with the by-product assumption.

To describe how Model (13) measure the unified efficiency related to Stage III (B) of Fig. 2, this study needs to show the dual formulation of Model (13) as follows:

$$
\begin{aligned}
& \text { Minimize }-\sum_{i=1}^{m} v_{i} x_{i k}+\sum_{r=1}^{s} u_{r} g_{r k}-\sum_{f=1}^{h} w_{f} b_{f k}+\sigma \\
& \text { s.t. } \quad-\sum_{i=1}^{m} v_{i} x_{i j}+\sum_{r=1}^{s} u_{r} g_{r j}-\sum_{f=1}^{h} w_{f} b_{f j}+\sigma \geq 0 \quad(j=1, \ldots, n) \text {, } \\
& v_{i} \geq \varepsilon R_{i}^{x} \\
& u_{r}: \text { URS } \\
& (i=1, \ldots, m) \text {, } \\
& (r=1, \ldots, s) \text {, } \\
& w_{f} \geq \varepsilon R_{f}^{b} \quad(f=1, \ldots, h) \& \\
& \sigma: \text { URS. }
\end{aligned}
$$

To specify the analytical implication of Model (14), this study needs to discuss Complementary Slackness Conditions (CSCs) between primal and dual models. The proposed proof may be a straightforward manner. That is, the CSCs between Models (13) and (14) contain the following equations:

$$
\left(-\sum_{i=1}^{m} v_{i} x_{i j}+\sum_{r=1}^{s} u_{r} g_{r j}-\sum_{f=1}^{h} w_{f} b_{f j}+\sigma\right) \lambda_{j}=0 \quad \text { for } \quad j=1, \ldots, n \text {. }
$$

A reference set for the $k$ th DMU in $\mathrm{RS}_{k}$ consists of DMUs that have $\lambda_{j}>0$ for $j \in \mathrm{RS}_{k}$. Therefore, the supporting hyperplane is determined by

$$
-\sum_{i=1}^{m} v_{i} x_{i j}+\sum_{r=1}^{s} u_{r} g_{r j}-\sum_{f=1}^{h} w_{f} b_{f j}+\sigma=0, \quad j \in \mathrm{RS}_{k} .
$$

The supporting hyperplane is expressed by $-v^{*} x+u^{*} g-w^{*} b+\sigma^{*}=0$ on an efficiency frontier in the case when three production factors have a single component. The simple case is for our descriptive convenience. The supporting hyperplane is expressed by $b=\left(-v^{*} x+u^{*} g+\sigma^{*}\right) / w^{*}$, where both $w^{*}$ and $v^{*}$ are positive, the slope of a supporting 
hyperplane is determined by $u^{*}$. If $u^{*}$ is positive, the amount of $g$ increases $g$, along with $b$, because $v^{*}$ is positive. If $u^{*}$ is negative, then the amount of $x$ decreases $b$, This situation indicates the right-hand side of Stage III (B) in Fig. 2. In contrast, if $u^{*}$ is positive, then the amount of $g$ increases $b$. This situation indicates the left-hand side of Stage III (B) in Fig. 2.

After solving Model (13), we can identify a possible occurrence of DC, or green technology innovation, by the previous rule along with the assumption on a unique optimal solution: This study can identify a possible occurrence of DC, or green technology innovation, by the following rule along with the assumption on a unique optimal solution:

1. if $u_{r}^{*}=0$ for some (at least one) $r$, then "weak DC" occurs on the $k$ th DMU,

2. if $u_{r}^{*}<0$ for some (at least one) $r$, then "strong DC" occurs on the $k$ th DMU and

3. if $u_{r}^{*}>0$ for all $r$, then "no DC" occurs on the $k$ th DMU.

Note that if $u_{r}^{*}<0$ for some $r$ and $u_{r^{\prime}}^{*}=0$ for the other $r^{\prime}$, then the weak and strong DCs coexist on the $k$ th DMU. We consider it as the strong DC, so indicating technology innovation on undesirable outputs. Here, it is important to add that $u_{r}^{*}<0$ for all $r$ is the best case because an increase in any desirable output always decreases an amount of undesirable outputs. Meanwhile, if $u_{r}^{*}<0$ is identified for some $r$, then it indicates that there is a chance to reduce an amount of undesirable output(s). Therefore, we consider the second case as an occurrence of DC. The three concerns (e.g., multiple solutions and slack adjustment) on UC are also applicable to DC. Finally, it is important to note that this section solves the second pitfall.

\section{Handling zero and negative values}

This study fully utilizes the property of "translation invariance" applied to Model (13) that is our new formulation. The property uses the following data shifts on all DMUs $(j=1, \ldots, n)$ :

$$
\tilde{x}_{i j}=x_{i j}+\alpha_{i}(i=1, \ldots, m), \tilde{g}_{r j}=g_{r j}+\beta_{r}(r=1, \ldots, s) \text { and } \tilde{b}_{f j}=b_{f j}+\delta_{f}(f=1, \ldots, h) \text { (17) }
$$

The three Greek symbols are specific positive numbers (e.g., 1 and 10) that are subjectively selected by a DEA user(s). As a result of these data shifts, all production and environmental factors of the $j$ th DMU become $\tilde{x}_{i j}>0(i=1, \ldots, m), \tilde{g}_{r j}>0(r=1, \ldots$, $s)$ and $\tilde{b}_{f j}>0(f=1, \ldots, h)$, where the symbol $(>)$ implies strict positivity in all components of the three vectors for production factors.

Here, it is necessary to note that the proposed data shifts can be applied to any data set within our computational common sense. For example, in the single component case, $\tilde{x}=x+100,000(i=1, \ldots, m), \tilde{g}=g+\beta(=1)(r=1, \ldots, s)$ and $\tilde{b}=b+\delta(=1)$ $(f=1, \ldots, h)$ are possible as the proposed data shifts (16). The shifts do not produce any mathematical difficulty to us. However, the large data (i.e., input) dominates the computational process Model (12) so often producing unreliable results. This is a computational problem, not a mathematical problem. 
Proposition The data shift, or $\tilde{x}_{i j}=x_{i j}+\alpha_{i}(i=1, . ., m), \tilde{g}_{r j}=g_{r j}+\beta_{r}(r=1, \ldots, s)$ and $\tilde{b}_{f j}=b_{f j}+\delta_{f}(f=1, \ldots, h)$, does not influence the unified efficiency measure under managerial disposability.

Proof To examine the property of translation invariance, let us return to Model (13) and modify the three groups of constraints as follows:

$$
\begin{aligned}
& \sum_{j=1}^{n}\left(x_{i j}+\alpha_{i}\right) \lambda_{j}-d_{i}^{x+}=x_{i k}+\alpha_{i}(i=1, \ldots, m), \sum_{j=1}^{n}\left(g_{r j}+\beta_{r}\right) \lambda_{j}=g_{r k}+\beta_{r}(r=1, \ldots, s) \\
& \text { and } \sum_{j=1}^{n}\left(b_{f j}+\delta_{f}\right) \lambda_{j}-d_{f}^{b}=b_{f k}+\delta_{f}(f=1, \ldots, h) .
\end{aligned}
$$

Equations (18) add a specific positive number to each production factor so that all observations become positive, shifting from zero or negative to positive, in their signs.

Equations (18) maintain the following three conditions or $\sum_{j=1}^{n} \alpha_{i} \lambda_{j}=\alpha_{i}$, $\sum_{j=1}^{n} \beta_{r} \lambda_{j}=\beta_{r}$ and $\sum_{j=1}^{n} \delta_{f} \lambda_{j}=\delta_{f}$, because of $\sum_{j=1}^{n} \lambda_{j}=1$. Consequently, Eq. (18) become

$$
\begin{aligned}
& \sum_{j=1}^{n} x_{i j} \lambda_{j}-d_{i}^{x-}=x_{i k}(i=1, \ldots, m), \sum_{j=1}^{n} g_{r j} \lambda_{j}=g_{r k}(r=1, \ldots, s) \text { and } \\
& \sum_{j=1}^{n} b_{f j} \lambda_{j}-d_{f}^{b}=b_{f k}(f=1, \ldots, h) .
\end{aligned}
$$

The above three groups of constraints are the same as those of Model (13). Thus, the proposed data shifts do not influence the constraints in Model (13).

Next, paying attention to the objective function of Model (13), the data shifts change it as follows:

$$
\begin{aligned}
& \sum_{i=1}^{m} \varepsilon R_{i}^{x}\left[\sum_{j=1}^{n}\left(x_{i j}+\alpha_{i}\right) \lambda_{j}-\left(x_{i k}+\alpha_{i}\right)\right]=\varepsilon \sum_{i=1}^{m} R_{i}^{x}\left(\sum_{j=1}^{n} x_{i j} \lambda_{j}-x_{i k}\right)=\varepsilon \sum_{i=1}^{m} R_{i}^{x} d_{i}^{x+} \text { and } \\
& \sum_{f=1}^{h} \varepsilon R_{f}^{b}\left[\sum_{j=1}^{n}\left(b_{f j}+\beta_{f}\right) \lambda_{j}-\left(b_{f k}+\beta_{f}\right)\right]=\varepsilon \sum_{f=1}^{h} R_{f}^{b}\left(\sum_{j=1}^{n} b_{f j} \lambda_{j}-b_{f k}\right)=\varepsilon \sum_{f=1}^{h} R_{f}^{b} d_{f}^{h} .
\end{aligned}
$$

Equation (20) clearly indicate the translation invariance in the objective value of Model (13). Thus, the proposed data shifts from zero and/or negative to positive do not influence the objective value of Model (13). The proposition indicates that the proposed approach can solve the last pitfall. 


\section{Conclusion and future extensions}

As an extension of the previous studies (e.g., Sueyoshi and Goto 2018) on DEA environmental assessment, this study discussed the methodological issues by considering theoretical and empirical difficulties related to the approach from sustainability development.

These difficulties included (a) how to incorporate two separated (natural and managerial) disposability concepts into the framework of DEA environment assessment, (b) how to reorganize unified disposability concepts for computation, (c) how to incorporate an occurrence of UC and that of DC and (d) how to manage a data set with zero and negative values. The newly proposed approach can overcome all of these methodological difficulties.

Here, we acknowledge that this study is not perfect. For example, this study has discussed how to measure the level of sustainability under DC, but not discussing these business implications on a scale benefit (e.g., RTS and DTS). The research concern will be discussed in future. Another research extension may be found in the incorporation of a time horizon into the proposed approach. This study has not explored such a research direction.

In conclusion, it is hoped that this study can contribute the DEA environmental assessment. We look forward to seeing future extension as discussed in this study.

Authors' contributions

TS identified four difficulties in DEA environmental assessment. TS and MG proposed concepts and models to solve the four difficulties in mathematical formulations. Both authors read and approved the final manuscript.

Author details

${ }^{1}$ Department of Management, New Mexico Institute of Mining and Technology, 801 Leroy Place, Socorro, NM 87801, USA. ${ }^{2}$ School of Environment and Society, Tokyo Institute of Technology, 2-12-1, Ookayama, Meguro-ku, Tokyo 152-8552, Japan.

\section{Acknowledgements}

We appreciate two anonymous reviewers for their careful reading of our manuscript and their insightful comments and suggestions. This work was supported by a Japan Society for the Promotion of Science (JSPS) Grant-in-Aid for Scientific Research (KAKENHI) 16K01236.

\section{Competing interests}

The authors declare that they have no competing interests.

Ethics approval and consent to participate

Not applicable.

\section{Publisher's Note}

Springer Nature remains neutral with regard to jurisdictional claims in published maps and institutional affiliations.

Received: 6 March 2018 Accepted: 20 May 2018

Published online: 16 July 2018

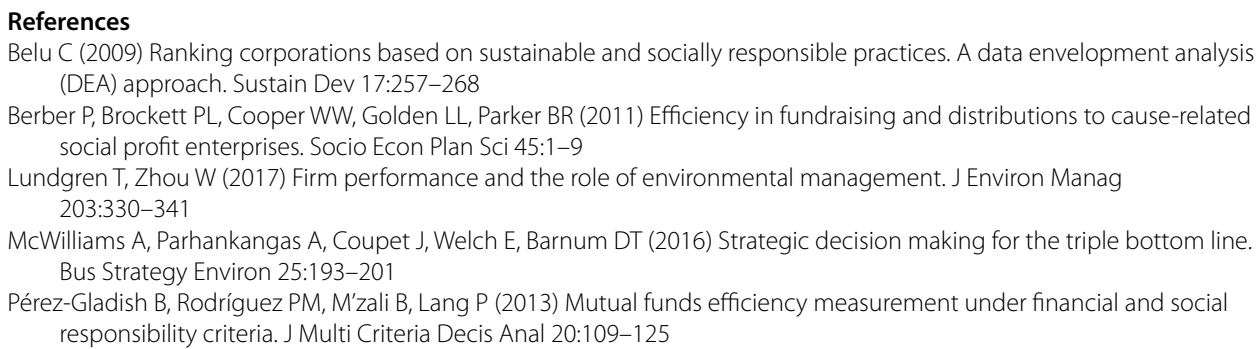


Sueyoshi T (2017) Damages to return with a possible occurrence of eco-technology innovation measured by DEA environmental assessment. J Econ Struct 6(7):1-14

Sueyoshi T, Goto M (2017) World trend in energy: an extension to DEA applied to energy and environment. J Econ Struct $6: 1-23$

Sueyoshi T, Goto M (2018) Environmental assessment on energy and sustainability by data envelopment analysis. Wiley, London, pp 1-609

Sueyoshi T, Yuan Y (2018) Measuring energy usage and sustainability development in Asian nations by DEA intermediate approach. J Econ Struct 7:1-18

Sueyoshi T, Goto M, Omi Y (2010) Corporate governance and firm performance: evidence from Japanese manufacturing industries after the lost decade. Eur J Oper Res 203:724-736

Sueyoshi T, Yuan Y, Goto M (2017) A literature study for DEA applied to energy and environment. Energy Econ 62:104-124

Vitaliano DF, Stella GP (2006) The cost of corporate social responsibility: the case of the community reinvestment act. J Prod Anal 26:235-244

Wang WK, Lu WM, Kweh QL, Lai HW (2014) Does corporate social responsibility influence the corporate performance of the U.S. telecommunications industry? Telecommun Policy 38(7):580-591

Yang SL (2016) Corporate social responsibility and an enterprise's operational efficiency: considering competitor's strategies and the perspectives of long-term engagement. Qual Quant 50:2553-2569

Yang FC (2017) Integrating corporate social responsibility and profitability into best practice selection: the case of large Taiwanese firms. Qual Quant 51:1493-1512

\section{Submit your manuscript to a SpringerOpen ${ }^{\circ}$ journal and benefit from:}

- Convenient online submission

- Rigorous peer review

- Open access: articles freely available online

- High visibility within the field

- Retaining the copyright to your article

Submit your next manuscript at $\gg$ springeropen.com 